'Servicio de Reumatología, Hospital Militar de Santiago. Facultad de Medicina Universidad de Valparaíso. Chile.

${ }^{2}$ Médico Becado, Medicina Interna, Facultad de Medicina Universidad del Desarrollo. Santiago, Chile.

${ }^{3}$ Servicio de Hemato-Oncología Hospital Militar de Santiago. Chile.

${ }^{4}$ Servicio de Anatomía Patológica, Hospital Militar de Santiago. Chile.

Recibido el 31 de julio de 2016, aceptado el 31 de agosto de 2016

Correspondencia a: Dra. Daniela Grünholz Gambi danielagru@gmail.com

\section{Arteritis de células gigantes: compromiso extenso de la aorta y grandes ramas demostrado por tomografía por emisión de positrones. Caso clínico}

DANIELA GRÜNHOLZ ${ }^{1}$, MARÍA PAZ POBLETE ${ }^{2}$ LORETO OVALLE ${ }^{1}$, EDUARDO WAINSTEIN ${ }^{1}$, GLORIA RUBIO ${ }^{3}$, MARÍA MANRÍQUEZ ${ }^{4}$, KARIN KALBHENN ${ }^{1}$

\section{Extensive arterial involvement in giant cell arteritis. Report of one case}

Large vessel vasculitis and particularly Temporal Arteritis are systemic diseases that may affect the aorta and its major branches, mainly involving extra cranial branches of the carotid artery. We report a 72-year-old man presenting with weight loss, fever and malaise. Positron emission computed tomography (PETCT) showed an extensive inflammation of the aorta and its major branches. Temporal artery biopsy confirmed the presence of vasculitis with granulomas. Treatment with a high dose of steroids had an excellent clinical response. This case underscores the utility of PET CT in the assessment of this disease.

(Rev Med Chile 2016; 144: 1486-1490)

Key words: Giant Cell Arteritis; Positron-Emission Tomography; Tomography; Tomography, Emission-Computed.

\section{L}

a arteritis de células gigantes o arteritis de la temporal es una vasculitis sistémica de etiología desconocida. Afecta principalmente a los grandes vasos, en especial a la arteria temporal, carótida, aorta y sus ramas. En países occidentales y en mayores de 50 años la incidencia se ha estimado en 200 casos por cada 100.000 personas, ,con un predominio en el sexo femenino.

La prevalencia y la incidencia de la vasculitis de células gigantes muestra que es una entidad subdignosticada ${ }^{1-8} \mathrm{y}$ dependen del método diagnóstico usado, la región geográfica y la sospecha clínica ${ }^{8}$.

Aunque la etiología de la enfermedad es desconocida, en su fisiopatología se ha demostrado el rol de IL-6, IL-1 e interferón. La producción de IL-1 por las células endoteliales puede activar los linfocitos T y tanto la IL-1 como el TNF alfa son potentes inductores de ELAM-1 (molécula 1 de adhesión endotelio-leucocito) y de VCAM-1 (molécula 1 de adhesión vascular), los que promueven la adhesión de los leucocitos desde la sangre a la pared vascular ${ }^{3}$.

La afección granulomatosa característica en la histología tiene predilección por las ramas craneales de las arterias que se originan en el cayado aórtico.

Las manifestaciones clínicas más frecuentes están dadas por el compromiso de las arterias inflamadas, especialmente en cabeza y cuello: amaurosis fugax, cefalea, claudicación mandibular. Los síntomas constitucionales también pueden estar presentes: baja de peso, compromiso del estado general, fiebre entre otros.

La complicación más grave se debe al compromiso isquémico de los diferentes territorios comprometidos y la amaurosis es la más frecuente 
y temida por el compromiso de la arteria oftálmica $^{1-6}$.

El diagnóstico y el tratamiento precoz pueden prevenir estas complicaciones, sin embargo, en ocasiones, el diagnóstico es difícil debido a que los síntomas y el estudio de laboratorio es inespecífico, describiéndose hasta $15 \%$ de pacientes en los cuales el laboratorio general es normal.

La biopsia de arteria temporal sigue siendo el gold standard para el diagnóstico, con una sensibilidad y especificidad que bordean 75 y 90\%, respectivamente 5 . Se describe un valor predictivo negativo de $90 \%{ }^{13}$. Por otro lado, no siempre hay afección de la arteria temporal.

Dentro de las técnicas de ayuda diagnóstica se encuentra la ecotomografía con Doppler de arteria temporal, que muestra una zona hipoecogénica que rodea la arteria temporal, que corresponde a edema del vaso. Este signo ecográfico, denominado signo del halo, ha demostrado alta especificidad, pero baja sensibilidad ${ }^{10}$.

El compromiso de la aorta y sus ramas ha sido subdiagnosticado, sin embargo, con el advenimiento de nuevas técnicas de imagen como la tomografía por emisión de positrones (PET)/ tomografía axial computada (PET/CT) ha aumentado su detección ${ }^{4-6}$.

El uso de PET en el diagnóstico de vasculitis de grades vasos ha sido refrendado en diversos estudios $^{2-4,6,7,11-13}$. La visualización por PET del aumento de captación de glucosa marcada en las paredes inflamadas de los vasos afectados es de gran importancia en el diagnóstico ${ }^{3}$. Esta técnica de medicina nuclear muestra que el compromiso inflamatorio de la aorta y subclavia puede estar presente hasta en 50 a $80 \%$ de los pacientes ${ }^{13}$.

A continuación se describe un caso clínico en el cual el paciente presenta un extenso compromiso de aorta y sus grandes vasos y la utilidad de PET en el diagnóstico.

\section{Caso clínico}

Paciente de 72 años, sexo masculino, previamente sano. Consultó por cuadro de 4 meses de duración caracterizado por cefalea holocránea, baja de peso de 10 kilos, gran compromiso del estado general, astenia, adinamia y sudoración nocturna.
Al examen se encontraba decaído, pálido y enflaquecido, con fuerza muscular conservada, sin sinovitis, rangos articulares conservados, sensibilidad en epigastrio, sin adenopatías, sin sensibilidad en arterias temporales a la palpación.

$\mathrm{Al}$ interrogatorio dirigido, refería en una ocasión amaurosis izquierda de 30 min de duración con resolución completa y, ocasionalmente, dolor mandibular frente a la masticación.

De su estudio destacaba: hematocrito de $32 \%$, hemoglobina de $10,2 \mathrm{~g} / \mathrm{dL}$, volumen corpuscular medio de 80fL (VR: 84-94), concentración de hemoglobina corpuscular media de $33 \mathrm{~g} / \mathrm{dL}$ (31-36). Velocidad de eritrosedimentación: $96 \mathrm{~mm} / \mathrm{h}$; leucocitos $6.200 \mathrm{x} \mathrm{mm}^{3}$, fórmula normal, plaquetas $440.000 \times \mathrm{mm}^{3}$; proteína C reactiva de $80 \mathrm{mg} / \mathrm{dl}$ (VR: hasta $5 \mathrm{mg} / \mathrm{dl}$ ); perfil bioquímico, lipídico, renal, hepático y tiroideo normal, orina completa normal, electroforesis de proteínas mostró hipergammaglobulinemia difusa leve.

Cinética de fierro: ferremia $16 \mathrm{ug} / \mathrm{dL}$ (VR: 50-170), capacidad total de fijación del hierro $224 \mathrm{ug} / \mathrm{dL}$ (VR: 250-450), transferrina $187 \mathrm{ug} /$ dL (VR: 210-420), saturación de hierro 7\%, (VR: 20-55) ferritina $450 \mathrm{ng} / \mathrm{dL}$ (VR: 22-322), factor reumatoideo negativo, niveles de complemento normal, resonancia magnética (RNM) de cerebro sin lesiones, colonoscopia normal, endoscopia digestiva alta: gastropatía fúndica de aspecto atrófico. Enteroscopia con cápsula endoscópica normal. Alfafetoproteína y antígeno carcinoembrionario, normales. Ecocardiograma mostró insuficiencia mitral leve.

Tomografía axial computada de tórax, abdomen y pelvis: normal. AngioTAC de vasos del cuello y tórax: presencia de ateromatosis mixta que predomina en el origen de la arteria subclavia izquierda y que no determina una estenosis significativa.

Dado cuadro sin etiología clara y alta sospecha de arteritis de células gigantes y para descartar cuadro de origen neoplásico, se decidió realizar tomografía por emisión de positrones, que informó: intensa hiperfijación difusa e irregular del trazador en paredes de la aorta ascendente, cayado, y descendente, bifurcación de ilíacas y femorales, así como subclavias, carótidas y arterias occipitales. Foco irregular de mayor concentración de fluorodesoxiglucosa (FDG) en región cervical baja anterior derecha de 2,3 cm. Conclusión: PET-FDG sin evidencias categóricas de actividad 


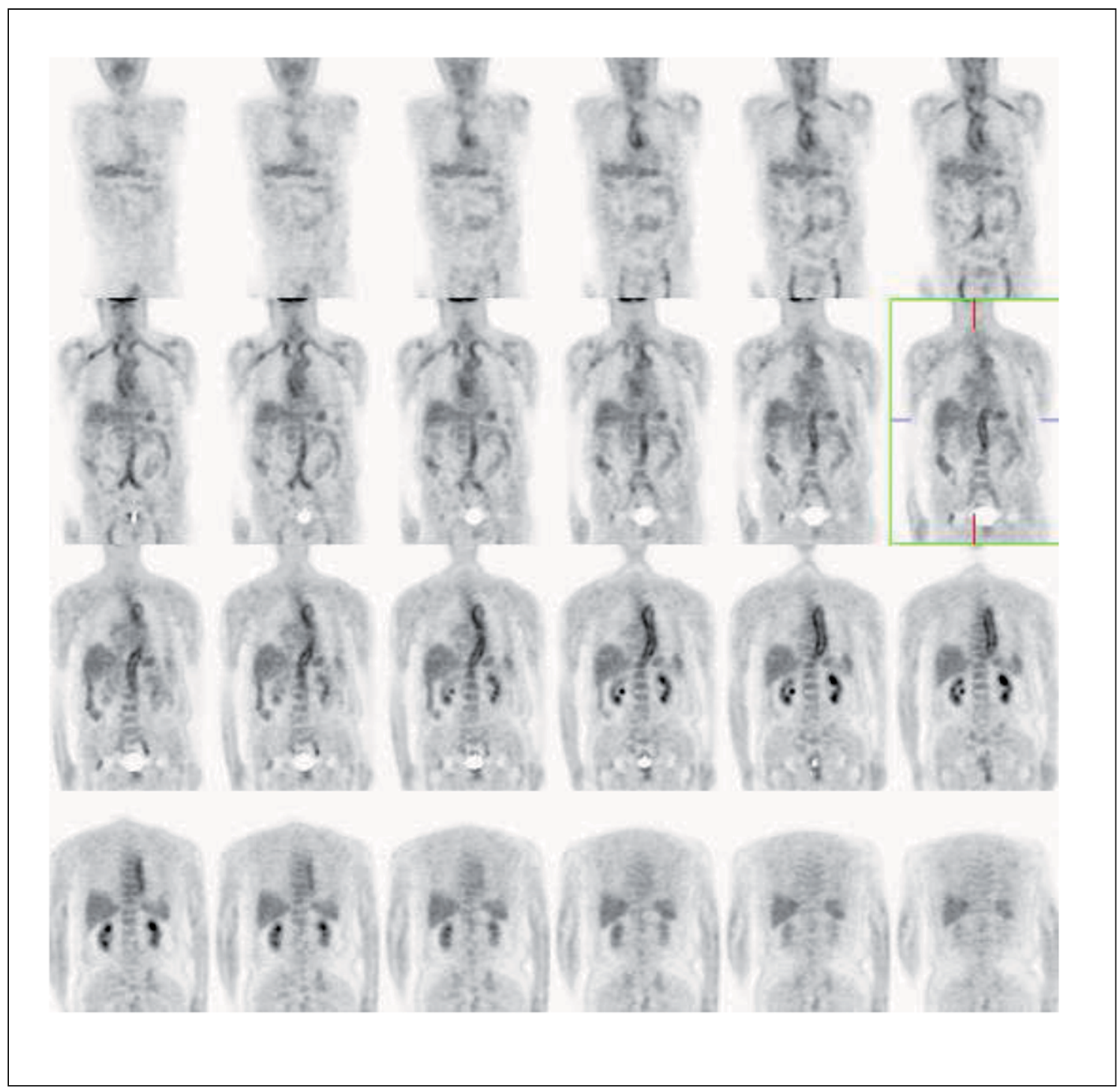

Figura 1. PET: intensa hiperfijación difusa e irregular del trazador en paredes de la aorta ascendente, cayado, y descendente, bifurcación de ilíacas y femorales, así como subclavias, carótidas y arterias occipitales.

hipermetabólica tumoral maligna. El hallazgo en paredes arteriales es sugerente de arteritis: el foco cervical hace necesario descartar nódulo tiroideo (Figura 1).

Ecografía tiroidea: tiroides heterogénea sugerente de tiroiditis crónica.

Dados los hallazgos anteriormente descritos se decidió iniciar prednisona $50 \mathrm{mg} /$ día $(1 \mathrm{mg} /$ $\mathrm{kg}$ ), vo. La biopsia de arteria temporal (obtenida una semana después de iniciado el tratamiento) mostró: vasculitis granulomatosa con algunas cé- lulas gigantes multinucleadas en la pared muscular alrededor de vasos, acompañados de linfocitos y algunos plasmocitos. (Figura 2).

El paciente luego de 3 días de iniciado el tratamiento refirió mejoría notable del compromiso del estado general.

El control desde el punto de laboratorio a las 2 semanas del inicio del tratamiento mostró descenso de la VHS a $20 \mathrm{~mm} / \mathrm{h}$, PCR a valores normales y el paciente realizando sus actividades de la vida cotidiana. 


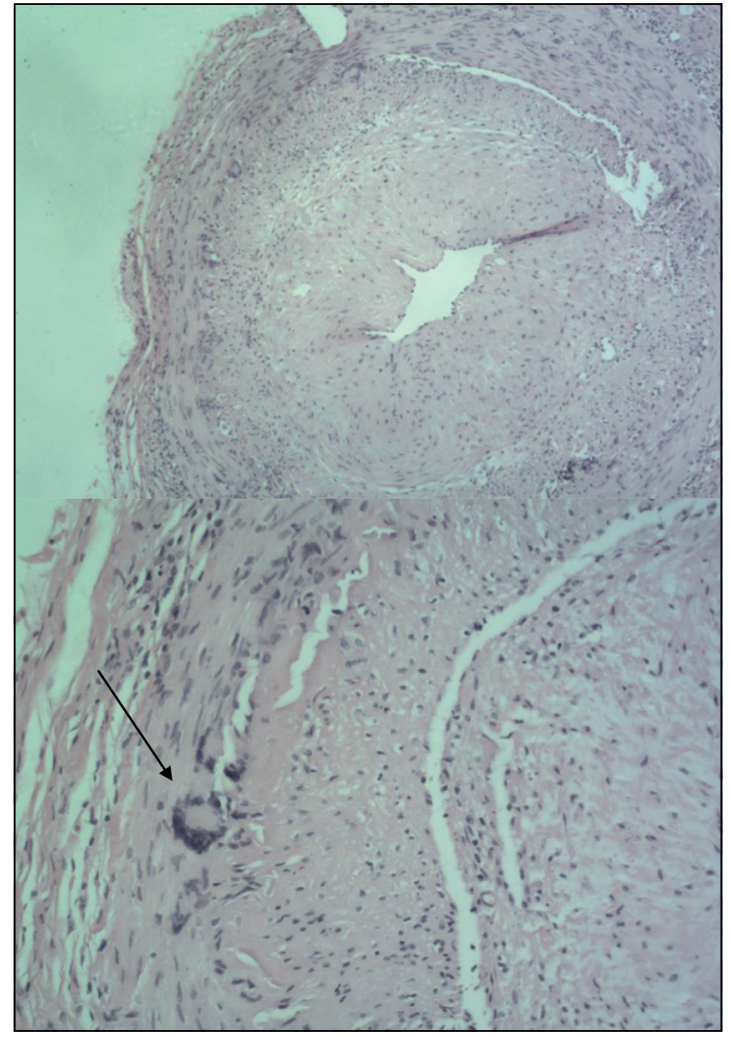

Figura 2. Imagen superior Hematoxilina/Eosina 10x. Corte transversal de arteria temporal con abundante infiltrado linfoplasmocitario en capa muscular. Imagen inferior: Hematoxilina /Eosina10x, la flecha muestra un granuloma.

\section{Discusión}

Previo al desarrollo de las técnicas de medicina nuclear, en la arteritis de células gigantes se describía un compromiso extracraneal que iba de 15 a 17\%, sin embargo, con esta herramienta diagnóstica, este compromiso se describe entre 30 y $74 \%{ }^{7-10}$.

El diagnóstico tardío del compromiso inflamatorio extracraneal de la enfermedad se ha asociado a complicaciones vasculares graves, que incluyen aneurismas y enfermedad vascular oclusiva ${ }^{8-11}$.

La técnica que usualmente se utiliza para diagnosticar esta entidad corresponde a la biopsia de la arteria temporal, sin embargo, es un procedimiento invasivo, tiene una sensibilidad de alrededor de $50 \%{ }^{10,11}$ y estudios recientes demuestran que la cefalea no necesariamente ayuda a guiar el sitio de la biopsia ${ }^{16}$. Por otro lado, el compromiso de los vasos torácicos, que está presente en $45 \%$ de los pacientes recientemente diagnosticados, se asocia con una biopsia de arteria temporal negativa hasta en $50 \%$ de los casos reportados ${ }^{10,11}$.

Las imágenes del PET revelan una mayor concentración de FDG en las paredes de vasos de $4 \mathrm{~mm}$ de diámetro o más, que traducen inflamación que precede a los cambios morfológicos en vasculitis ${ }^{7,8}$, incluso, hay autores que recomiendan el uso de esta técnica de medicina nuclear a pesar de que no se encuentra en los criterios diagnósticos $^{9-11}$.

Se estima que el PET tiene una sensibilidad entre 56 y $100 \%$ en el diagnóstico de vasculitis de células gigantes ${ }^{7}$.

Prieto-González y cols ${ }^{7}$ demostraron que la captación de glucosa marcada en sujetos con vasculitis previamente diagnosticada con biopsia fue significativamente mayor que en sujetos sanos.

Dentro de los diagnósticos diferenciales a considerar frente a un PET positivo para inflamación vascular se debe considerar la enfermedad ateroesclerótica severa, la aortitis idiopática, la enfermedad relacionada a IgG4 y la aortitis sifilítica ${ }^{13}$.

Dentro de las limitaciones principales de esta técnica se describe la heterogénea estandarización en la definición de parámetros objetivos de inflamación de los vasos y en la variabilidad interobservador ${ }^{13}$. En este sentido, la revisión sistemática realizada por Puppo y cols ${ }^{10}$ menciona que existen métodos cualitativos, semicuantitativos y combinados para evaluar la captación de glucosa en las paredes de los vasos $y$, a pesar que faltan estudios al respecto, los autores concluyen que aparentemente los métodos cualitativos (escalas visuales de Blockmans, Mellery Brodmann) serían más específicos, pero menos sensible que los métodos cuantitativos $^{7,12-15}$.

Otra de las limitaciones de esta técnica es que no permite evaluar los vasos craneales, es decir, no es un examen de elección para evaluar el compromiso de arterias temporales ${ }^{7-16}$, por otro lado, su alto costo hace que no esté disponible en todos los centros en Chile.

Las ventajas del PET en el diagnóstico de vasculitis de células gigantes es que puede diagnosticar estadíos muy tempranos de la enfermedad, previos a los cambios morfológicos irreversibles, es una técnica no invasiva, y a pesar que la biopsia sigue siendo el gold standard para el diagnóstico, es una 
herramienta útil en un grupo de pacientes que pueden presentarse como casos atípicos, pacientes con historia de fiebre de origen desconocido, o síndrome inflamatorio sin explicación ${ }^{16-19}$.

\section{Conclusión}

Este caso clínico demuestra que en las vasculitis de vaso grande puede haber un extenso compromiso vascular inflamatorio asintomático u oligosintomático y, por otro lado, demuestra la utilidad del PET en el diagnóstico y tratamiento oportuno, herramienta útil especialmente en pacientes adultos mayores con fiebre de origen desconocido.

\section{Referencias}

1. Klein RG, Hunder G, Stanson A, Stanson and Sheps G. Large artery involvement in giant cell (temporal) arteritis. Ann Inter Med 1975; 83: 806-12.

2. Bogaard K, Schrama J, Voogel, A, Zwijnenburg A, Ezechiels J. A Detection of Giant Cell Aortitis Using 18F-Fluorodeoxyglucose Positron Emission Tomography Computed Tomography. Circulation 2010; 122: 411-2.

3. Villa I, Agudo M, Martínez-Taboada V. Avances en el diagnóstico de las vasculitis de vasos de gran calibre: identificación de biomarcadores y estudio de imagen. Reumatol Clin 2011; 7 (S3): S22-7.

4. Pacheco M, Mínguez M, Martínez A, Bernabeu M. Diagnóstico precoz de las vasculitis de grandes vasos. Utilidad de la tomografía por emisión de positrones con la tomografía axial computada. Reumatol Clin 2013; 9 (1): 65-8.

5. Kesten F, Aschwanden M, Gubser P, Glatz K, Daikeler T, Hess C. Giant cell arteritis a changing entity. Swiss MedWkly 2011; 141: w13272.

6. Bruls S, Courtois A, Namur G, Smeets JP, Nusgens B, Michel JB, et al. Increased Metabolic Activity Highlighted by positron emission tomography/computed tomography in the wall of dissected ascending aorta in a patien with Horton disease. Circ Cardiovasc Imaging 2013; 6: 606-8.

7. Prieto-González S, Depetris M, García-Martínez A, Espigol-Frigolé G, Tavera-Bahillo I, Corvera-Bellata M, et al. Positron emission tomography assessment of large vessel inflammation in patients with newly diagnosed, biopsyproven giant cell arteritis: a prospective, case-control study. Ann Rheum Dis 2014; 73: 1388-92.
8. Balink H, Bennink R, van Eck-Smit B, Verberne H. The role of F-FDG PET/CT in large-vessel vasculitis: appropriateness of current Classification Criteria? Bio Med Research International 2014; ID687608.

9. Berti A, Campochiaro C, Cavalli G, Pepe G, Praderio L, Sabbadini MG, et al. Giant cell arteritis restricted to the limb arteries: An overlooked clinical entity. Autoimmun Rev 2015; 14 (4): 352-7.

10. Puppo C, Massollo M, Paparo F, Camellino D, Piccardo A, Shoushtari Zadeh Naseri M, et al. Giant Celles Arteritis: A Systematic Review of the qualitative and Semiquantitative Methods to asses vasculitis with 18F-Fluorodeoxyglucose positrón Emission Tomography. Biomed Res Int 2014; 2014: 574248.

11. Vega R, Morales F, Kumar S. Are we missing diagnosis of large vessel vasculitis? Role of F-Fluorodeoxyglucose PET-CT. Am J Med 2015; 128 (7): e15-6.

12. Ponte C, Rodrigues A, O’Neill L, Luqmani R. Giant cell Arteritis. Current Treatment and management. World J Clin Cases 2015; 3 (6): 484-94.

13. Lensen K, Comans E, Voskuyl A, van der Laken C, Brouwer E, Zwijnenburg A, et al. Large-vessel vasculitis: interobserver Agreement and Diagnostic Accuracy of 18F-FDG-PET/CT. Biomed Res Int 2015; 2015: 914692.

14. Soussan M, Nicolas P, Schramm C, Katsahian S, Pop G, Fain O, et al. Management of Large-Vessel Vasculitis with FDG-PET. Medicine (Baltimore) 2015; 94 (14): e622.

15. Stellingwerff MD, Brouwer E, Lensen KJ, Rutgers A, Arends S, van der Geest KS, et al. Different Scoring Methods of FDG PET/CT in Giant Cell Arteritis: Need for Standardization. Medicine (Baltimore) 2015; 94 (37): e1542.

16. Brodmann M, Lipp R, Passath A, Seinost G, Pabst E, Pilger E. The role of 2 F-fluoro-2-deoxy-D-glucose positrón emission tomography in the disgnosis of giant cell arteritis of the temporal arteries. Reumathology 2004; 43: 241-2.

17. Blockmans D. Diagnosis and extension of giant cell arteritis. Contribution of imaging techniques. Presse Med 2012; 41: 948-54.

18. Brückner M, Bettenworth D, Hengst $K$, Weckesser M, Willeke P, Heidemann J. Giant cell arteritis exclusively detected by $18 \mathrm{~F}$-fluorodeoxyglucose positron emission tomography: a case report. J Med Case Rep 2014; 8: 356.

19. Kermani T, Warrington K, Cuthbertson D, Carette S, Hoffman G, Khalidi A, et al. Large-Vessel Involvement in Giant Cell Arteritis: A Population-Based Cohort Study of the Incidence-Trends and Prognosis. Ann Rheum Dis 2013; 72 (12): 1989-94. 